\title{
Suppression of the TeV pair-beam plasma instability by a weak intergalactic magnetic field
}

\author{
Mahmoud Alawashra ${ }^{a, *}$ and Martin Pohl ${ }^{a, b}$ \\ a Institute for Physics and Astronomy, University of Potsdam, \\ D-14476 Potsdam, Germany \\ ${ }^{b}$ DESY, \\ Platanenallee 6, D-15738 Zeuthen, Germany \\ E-mail: alawashra1@uni-potsdam.de, martin.pohl@desy.de
}

Several gamma-ray observations from distant blazars show a suppressed emission of the inverse Compton scattering cascade of the blazar-induced pair beams at the $\mathrm{GeV}$ energy band. There are two possible explanations, the first one is the deflections of the pair beam electrons and positrons by magnetic fields in the intergalactic medium. The second one is the drain of the pair energy by beam-plasma instabilities resulting in heating up the intergalactic plasma. The studies of plasma instabilities of blazar-induced pair beams in the literature neglect the effect of weak intergalactic magnetic fields. In this work, we investigate the effect of weak intergalactic magnetic fields with small correlation lengths on the electrostatic beam-plasma instability. Such weak fields do not modify the dispersion relation describing the electrostatic waves and so the linear growth rate description. However, We found that the increase of the angular distribution of the particles of the pair beam due to the weak intergalactic fields reduces the linear growth rate of the electrostatic instability. This reduction of the linear growth rate increases the energy loss time of the beamplasma instability suppressing it against the inverse Compton scattering at magnetic field strengths three orders of magnitude less than the lower limit of the magnetic fields needed to deflect the secondary cascade emission.

$37^{\text {th }}$ International Cosmic Ray Conference (ICRC 2021)

July 12 th - 23rd, 2021

Online - Berlin, Germany

\footnotetext{
${ }^{*}$ Presenter
} 


\section{Introduction}

Various GeV-TeV high energy gamma rays from distant blazars $(z>0.024)$ have been observed by the Fermi-LAT telescope and the imaging atmospheric Cerenkov telescopes (i.e VERITAS, MAGIC and HESS) [2,8]. Very high energy gamma rays $\left(E_{\gamma}>1 \mathrm{TeV}\right)$ annihilate with the extra-galactic background light (EBL) producing electron-positron pairs, that are expected to lose their energies via the inverse Compton scattering on the cosmic microwave background (CMB) over a distance $\lambda_{\mathrm{IC}} \approx(75 \mathrm{kpc}) \frac{10^{7}}{\gamma_{b}}$ producing an electromagnetic cascade in the $\mathrm{GeV}$ energy band. Nevertheless, some of the observed blazars arriving energy fluxes in the $\mathrm{GeV}$ band are less than the predicted flux of the full electromagnetic cascade [10].

One possible explanation for the absence of the GeV secondary flux is that the electrons and the positrons of the beam had been deflected significantly in the intergalactic medium by fG-scale magnetic fields $[1,11,13]$. Another possibility is the beam-plasma electrostatic instability which works as an alternative energy loss mechanism to the inverse Compton scattering [5]. This instability builds up unstable electrostatic waves which through their nonlinear evolution drive away from the beam particles' kinetic energy heating up the intergalactic medium. The studies of the plasma instabilities of blazar-induced pair beams in the literature neglect the effect of the weak intergalactic magnetic fields on plasma instability. Here, We investigate the effect of weak intergalactic medium magnetic fields on the beam-plasma electrostatic instability.

We considered weak intergalactic magnetic fields with strengths of $B_{\mathrm{IGM}}<10^{-9}$ Gauss and with small correlation lengths that don't modify the linear dispersion relation of the beam-plasma instability. We showed that those magnetic fields lead the electrons and the positrons to perform a random walk passing through many correlation lengths with different magnetic field orientations resulting in increasing the angular spread of the beam particles that reduces the linear electrostatic instability growth rate significantly. The reduction of the linear growth rate increases the energy loss time of the beam-plasma instability which eventually becomes less than the energy loss time of the inverse Compton scattering suppressing the beam instability after a certain limit in the parameter space $\left(B_{\mathrm{IGM}}, \lambda_{\mathrm{B}}\right)$. This limit is found to be in a magnetic fields strength around three orders of magnetite less than the typical lower limit of the time delay of the arriving photons succeeding an interval of the source activity in the $\mathrm{TeV}$ band (around 10 years) [1].

\section{Electrostatic instability for a pair beam with weak intergalactic magnetic fields}

In this section, we investigate the effect of weak intergalactic magnetic fields with small correlation lengths $\left(\lambda_{B}<<\lambda_{\mathrm{IC}}\right)$ on the linear growth rate of realistic blazar-induced pair beam unstable electrostatic waves. Such weak magnetic fields don't change the linear dispersion relation description of the electrostatic instability as long as the background electrons gyromagnetic frequency $\omega_{B}=\frac{e B_{\mathrm{IGM}}}{m_{e}}$ is much smaller than their plasma frequency $\omega_{p}=\left(4 \pi n_{e} e^{2} / m_{e}\right)^{1 / 2}\left(\omega_{B}<<\omega_{p}\right)$ $[4,6]$. This is satisfied for intergalactic magnetic fields of strengths smaller than nano-Gauss.

However, we show in this section that the weak intergalactic magnetic fields impact the linear growth rate of the electrostatic instability through the significant dependence of the linear growth rate on the pair beam distribution function. Where the weak intergalactic magnetic fields with small correlation lengths increase the angular spread of the pair beam. Here we take the intergalactic 
background electrons temperature to be $T_{e}=10^{4} \mathrm{~K}$, their number density to be $n_{e}=10^{-7} \mathrm{~cm}^{-3}$ and the density number of the pair beam to be $n_{b}=3 \times 10^{-22} \mathrm{~cm}^{-3}$.

The linear growth rate of the unstable electrostatic waves due to a relativistic electron beam $\left(\gamma_{b}>>1\right)$ with a small angular spread $(\Delta \theta \leq 1 \mathrm{rad})$ traveling in a homogeneous background plasma with a density number $n_{e}$ is ([3])

$$
\omega_{i}(k)=\pi \omega_{p} \frac{n_{b}}{n_{e}}\left(\frac{\omega_{p}}{k c}\right)^{3} \int_{\theta_{1}}^{\theta_{2}} d \theta \frac{-2 g(\theta) \sin \theta+\left(\cos \theta-\frac{k c}{\omega_{p}} \cos \theta^{\prime}\right) \frac{\partial g(\theta)}{\partial \theta}}{\left[\left(\cos \theta_{1}-\cos \theta\right)\left(\cos \theta-\cos \theta_{2}\right)\right]^{1 / 2}}
$$

where

$$
g(\theta)=m_{e} c \int_{0}^{\infty} d p p f_{b}(p, \theta)
$$

and

$$
\cos \theta_{1,2}=\frac{\omega_{p}}{k c}\left(\cos \theta^{\prime} \pm \sin \theta^{\prime} \sqrt{\left(\frac{k c}{\omega_{p}}\right)^{2}-1}\right),
$$

where $k=\sqrt{k_{\perp}^{2}+k_{\|}^{2}}$ is the module of the unstable electrostatic waves wave-number vector $\left(k_{\perp}\right.$ and $k_{\|}$are the perpendicular and the parallel components to the beam propagation direction respectively), $\theta^{\prime}$ is the angle between the unstable electrostatic waves wave-number vector and the beam propagation direction, $\omega_{p}=\left(4 \pi n_{e} e^{2} / m_{e}\right)^{1 / 2}$ is the plasma frequency of the background electrons, $m_{e}$ is the electron mass, and $c$ is the speed of light.

The momentum distribution function $f_{b}(p, \theta)$ of the beam is normalized as follows

$$
2 \pi \int_{0}^{\infty} d p p^{2} \int_{0}^{\pi} d \theta \sin \theta f_{b}(p, \theta)=1
$$

and can be written as

$$
f_{b}(p, \theta)=f_{b, p}(p) f_{b, \theta}(p, \theta),
$$

where for the momentum distribution $f_{b, p}(p)$ we used the approximation obtained in [14] ( eq(26) and eq(56) in [14]) for realistic pair beam at distance $50 \mathrm{Mpc}$ from blazar.

Where for the angular distribution $f_{b, \theta}(p, \theta)$ we consider Gaussian angular spread due to the energy spread of the beam as in [9] along with Gaussian angular spread due to intergalactic magnetic fields with small correlation length, where such fields lead to stochastic deflections of the electrons and positrons increasing the angular distribution function of the pair beam through a Gaussian also

$$
f_{b, \theta}(\theta, p)=\frac{1}{\pi \Delta \theta^{2}} \exp -\left(\frac{\theta}{\Delta \theta}\right)^{2}, 0 \leq \theta \leq \pi, 0 \leq \varphi \leq 2 \pi,
$$

where

$$
\Delta \theta=\frac{m_{e} c}{p}\left(1+\sqrt{\frac{2}{3} \lambda_{B} \lambda_{\mathrm{IC}}} \frac{e B_{\mathrm{IGM}}}{m_{e} c}\right) .
$$

Finally, substituting eq(7) and eq(6) into eq(5) and eq(1) we found numerically the 2D spectrum of linear electrostatic waves growth rate for each value of the intergalactic magnetic field strength $B_{\mathrm{IGM}}$ and it's coloration length $\lambda_{B}$. The major effect of the intergalactic magnetic field on the linear growth rate is the reduction of the maximum growth rate as the intergalactic magnetic field strength and its correlation length increase as shown in Fig.1. This reduction in the growth rate increases the energy loss time of the beam-plasma instability as we will see in the next section. 


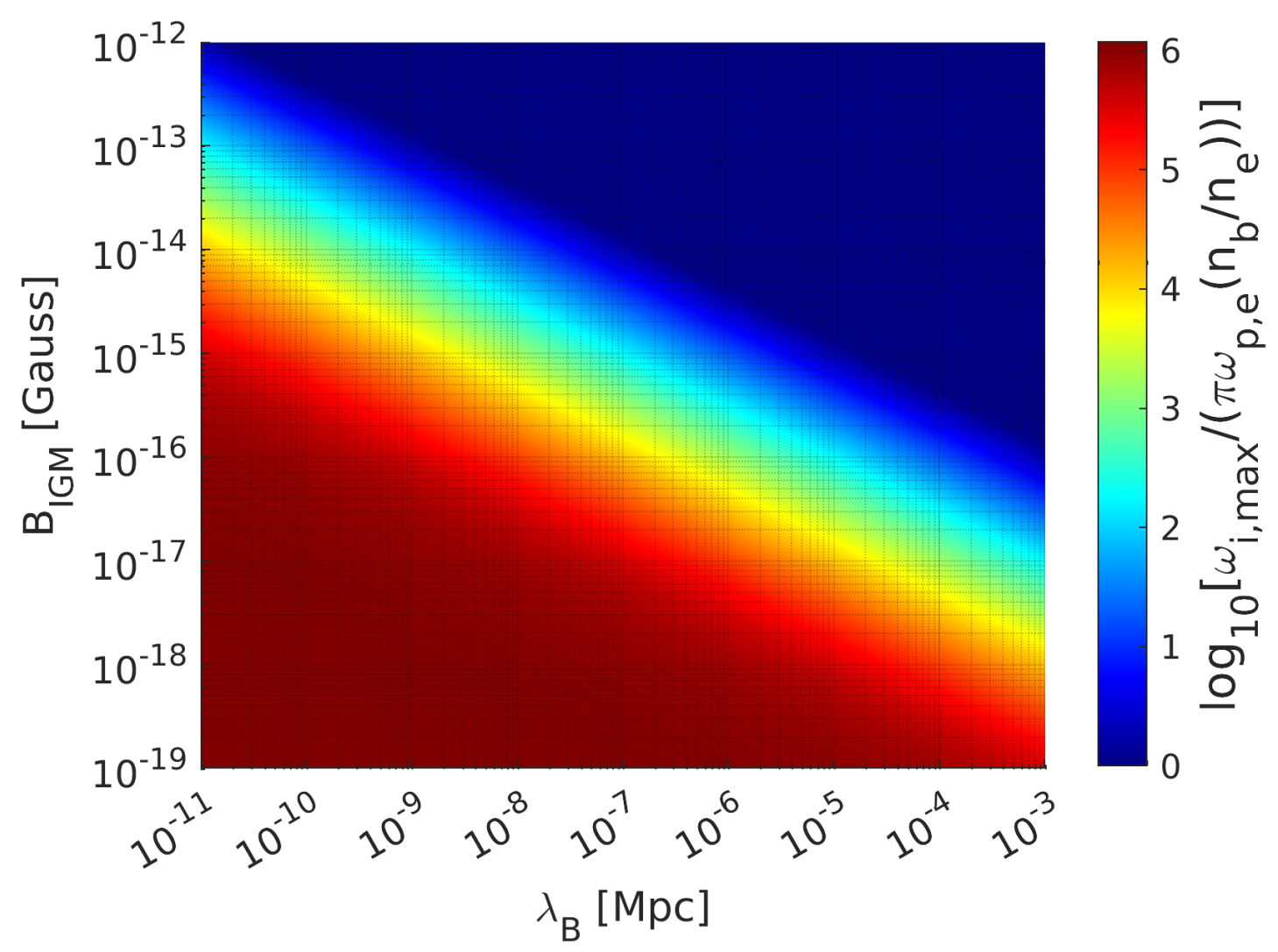

Figure 1: The logarithm of the maximum normalized linear growth rate $\log _{10}\left(\omega_{i, \max } /\left(\pi \omega_{p, e}\left(n_{b} / n_{e}\right)\right)\right)$.

\section{Nonlinear instability saturation}

The linear electrostatic growth rate excites the unstable electrostatic waves that depending on their nonlinear interactions could drain the kinetic energy of the pair beam heating up the background plasma of the intergalactic medium. The first type of those non-linear interactions is the scatterings of the unstable electrostatic waves on the background plasma particles known as the nonlinear Landau interactions. The second nonlinear interaction is the wave-wave interaction between the electrostatic waves known as modulation instability. The first process occurs at any wave energy intensity where the second occurs only above a certain threshold.

There are different estimations based on simulations and analytical calculations for the nonlinear evolution of the electrostatic waves which resulted in different values for energy loss time of the pair beam-plasma instability in the literature [5, 9, 12, 14]. However for this work, we consider the beam energy loss due to the plasma instability given in [14]

$$
\tau_{\text {loss }}=5 \times 10^{4} \omega_{i, \max }^{-1},
$$

where $\omega_{i, \max }$ is the maximum linear electrostatic growth rate in $\mathrm{Sec}^{-1}$ which is around $2.05 \times 10^{-7}$ $\mathrm{Sec}^{-1}$ when the intergalactic magnetic field is zero [14]. This time is needed to be compered with the energy loss time of the inverse Compton scattering to determine if the beam-plasma instability could 
suppress the $\mathrm{GeV}$ cascade emission in the intergalactic medium. The inverse-Compton scattering at red-shift $z=0.2$ and pair beam Lorentz factor $\gamma_{b}=4 \times 10^{6}$ is given by

$$
\tau_{\mathrm{IC}}=\left(1.3 \times 10^{-14} \mathrm{Sec}^{-1} \gamma_{b 6}(1+z)^{4}\right)^{-1}
$$

which gives

$$
\frac{\tau_{\text {loss }}}{\tau_{\mathrm{IC}}}=0.15
$$

when the intergalactic magnetic field is zero. However, the weak intergalactic magnetic fields increase the angular spread of the pair beam which reduces the linear electrostatic growth rate as we shown in the previous section. This reduction in the linear growth rate increases the energy loss of the beam-plasma instability as given by eq(8) which suppress the beam-plasma instability against the inverse Compton scattering after the limit where the energy loss of the beam-plasma instability is equal to the inverse Compton scattering energy loss time. We will demonstrate this limit in the next section.

\section{Results}

We found the maximum linear growth rate of the unstable electrostatic 2D spectrum for each intergalactic magnetic field strength $B_{\mathrm{IGM}}$ and correlation length $\lambda_{B}$ as shown in Fig.1. Then we calculated the approximated beam loss time of the beam-plasma instability based on the maximum linear growth rate as in eq(8). We compared then the beam energy loss time of the beam-plasma instability with the energy loss time of the inverse-Compton scattering at red-shift $z=0.2$ and pair beam Lorentz factor $\gamma_{b}=4 \times 10^{6}$ then we found the contour where the inverse Compton scattering time is equal to the plasma instability saturation time $\tau_{\mathrm{IC}}=\tau_{\text {loss }}$ (the purple line in Fig.2), above this line in the parameters space $\left(B_{\mathrm{IGM}}, d_{B}\right)$ the inverse Compton scattering acts on the pair beam faster than the plasma instability and so the plasma instability doesn't work as a sufficient energy loss mechanism of the beam in this region.

Finally, we compared in Fig. 2 the limit where the beam-plasma instability is suppressed against the inverse Compton scattering (the purple line) with the lower limit on the intergalactic magnetic field from the time-delayed of the arriving flux due to the deflection of the beam positrons and electrons by the intergalactic magnetic field (the green line) $[1,7,13]$. The Fermi (2018) lower limit [1] in Fig. 2 assumes an activity time of 10 years $\left(t_{\text {source }}=10 \mathrm{yr}\right)$ of the original source, and so the suppression of the cascade signal, in this case, is due to the time delay of the cascade emission after this activity time. Also, the opening jet angle has been set to $\theta_{\text {jet }}=6^{\circ}$ in Fermi (2018) analysis.

In Summery, in the intermediate region between the Fermi (2018) lower limit and the beamplasma instability limit in Fig.2, neither the beam-plasma instability nor the intergalactic magnetic field deflection work as an explanation for the observed blazars spectra and so this parameter space region can be excluded if there is no third mechanism that prevents the full electromagnetism cascade emission of the $\mathrm{TeV}$ gamma-ray beams from the distance blazars.

\section{Conclusion}

We investigated the effects of weak intergalactic magnetic fields with small correlation lengths on the blazar-induced pair beam electrostatic instability. The weak intergalactic magnetic fields 


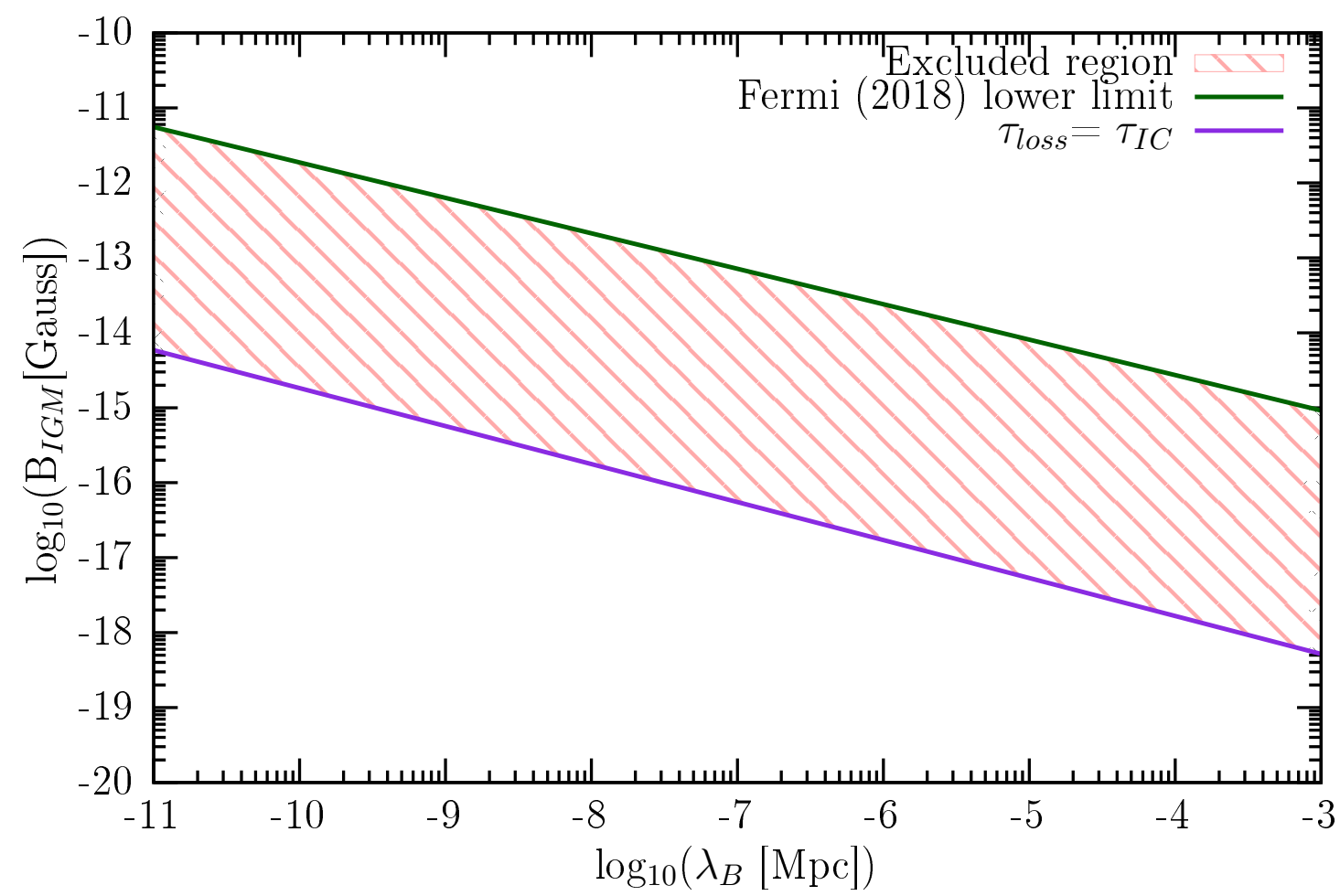

Figure 2: The intermediate excluded region of the intergalactic magnetic fields between the lower limit on the intergalactic magnetic field due to the time delay assuming blazar activity time of 10 years from Fermi observations (the green line) and the limit where plasma instability is suppressed (the purple line $\tau_{\text {loss }}=\tau_{\text {IC }}$ ).

don't change the linear dispersion relation description of the electrostatic instability but increase the angular spread of the pair beam distribution decreasing the linear growth rate of the electrostatic beam-plasma instability as the magnetic fields strength and correlation lengths increase. The reduction of the linear electrostatic growth rate decreases the energy loss time of the beam-plasma instability reaching values less than the inverse Compton scattering energy loss time after a certain limit. This limit is found to be 3 orders of magnitude smaller than the strength of the magnetic fields needed to deflect the cascade emission as shown in Fig.2. Finally, we excluded the parameter region between the instability limit and the time-delayed lower bound on the intergalactic magnetic field in Fig. 2 since neither the plasma instability nor the intergalactic magnetic field deflection work as an explanation for the observed blazars spectra in this region.

\section{Acknowledgement}

This work was supported by the International Helmholtz-Weizmann Research School for Multimessenger Astronomy, largely funded through the Initiative and Networking Fund of the Helmholtz Association. 


\section{References}

[1] Ackermann, M., Ajello, M., Baldini, L., et al. 2018, The Astrophysical Journal Supplement Series, 237, 32, doi: 10.3847/1538-4365/aacdf7

[2] Albert, J., Aliu, E., Anderhub, H., et al. 2008, Science, 320, 1752, doi: 10.1126/science. 1157087

[3] Breizman, B. 1990, Reviews of Plasma Physics, 15

[4] Brejzman, B., \& Ryutov, D. 1974, Nuclear Fusion, 14, 873-907, doi: 10 . 1088/0029-5515/ $14 / 6 / 012$

[5] Broderick, A. E., Chang, P., \& Pfrommer, C. 2012, The Astrophysical Journal, 752, 22, doi: $10.1088 / 0004-637 x / 752 / 1 / 22$

[6] Fainberg, Y. B. 1962, Journal of Nuclear Energy. Part C, Plasma Physics, Accelerators, Thermonuclear Research, 4, 203, doi: 10.1088/0368-3281/4/3/309

[7] Finke, J. D., Reyes, L. C., Georganopoulos, M., et al. 2015, The Astrophysical Journal, 814, 20, doi: $10.1088 / 0004-637 x / 814 / 1 / 20$

[8] H. E. S. S. Collaboration, Abramowski, A., Acero, F., et al. 2010, , 520, A83, doi: 10.1051/ 0004-6361/201014484

[9] Miniati, F., \& Elyiv, A. 2013, The Astrophysical Journal, 770, 54, doi: 10 . 1088/0004-637x/ $770 / 1 / 54$

[10] Neronov, A., \& Semikoz, D. V. 2009, , 80, 123012, doi: 10.1103/PhysRevD . 80 . 123012

[11] Neronov, A., \& Vovk, I. 2010, Science, 328, 73, doi: 10.1126/science. 1184192

[12] Schlickeiser, R., Ibscher, D., \& Supsar, M. 2012, The Astrophysical Journal, 758, 102, doi: $10.1088 / 0004-637 x / 758 / 2 / 102$

[13] Taylor, A. M., Vovk, I., \& Neronov, A. 2011, , 529, A144, doi: 10.1051/0004-6361/ 201116441

[14] Vafin, S., Rafighi, I., Pohl, M., \& Niemiec, J. 2018, The Astrophysical Journal, 857, 43, doi: $10.3847 / 1538-4357 /$ aab552 